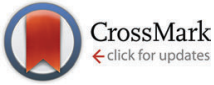

Cite this: Chem. Commun., 2016, 52,4521

Received 17th December 2015

Accepted 26th February 2016

DOI: $10.1039 / \mathrm{c} 5 \mathrm{cc} 10380 \mathrm{k}$

www.rsc.org/chemcomm

\section{Photoswitchable basicity through the use of azoheteroarenes $\dagger$}

\author{
Claire E. Weston, Robert D. Richardson and Matthew J. Fuchter*
}

\begin{abstract}
Azoheteroarene photoswitches offer functional advantages over their more conventional azobenzene counterparts by virtue of their heteroaromatic ring(s). Here we report that azobis(2-imidazole) functions as a photoswitchable base due to the additional proton stabilisation that is possible in the protonated $Z$ isomer, facilitated by the basic imidazole nitrogens. This thermodynamic difference in stability corresponds to a 1.3 unit difference in $\mathrm{p} K_{\mathrm{a}}$ values between the $E$ and $Z$ isomers. This $\mathrm{p} K_{\mathrm{a}}$ difference can be used to reversibly control solution $\mathrm{pH}$.
\end{abstract}

Using light as a means of modulating a system is extremely attractive, as it is non-invasive and is capable of providing excellent spatial and temporal precision. As such, numerous attempts have been reported that aim to use light to control a huge range of processes, from photopharmacology and optochemical genetics to spin-state switching and optomechanical devices; all mediated by photoresponsive molecules. ${ }^{1-7}$ One way in which light can be used to perturb a system is to modulate $\mathrm{pH}$. This opportunity can, for example, lead to external control of the reaction rate of pH-dependent chemical/biochemical processes; ${ }^{8-10}$ however thus far this is limited by the availability of suitable photoresponsive acids or bases. The change in acidity/basicity of a photoactive molecule could be an irreversible process, with examples reported for the release of a 'caged' proton upon irradiation with light. ${ }^{10,11}$ Alternatively (and preferably), the process could be reversible, through use of a suitable photoswitchable acid or base., ${ }^{8,912-16}$

Perhaps unsurprisingly, given their privileged nature in this field (synthetic versatility, high extinction coefficients and quantum yields, high fatigue resistance, etc.), a number of the photoswitchable acids and bases reported are azobenzenes, ${ }^{8,9,14}$ which can be reversibly switched between the thermally stable $E$ and metastable $Z$ isomers. Previously, differences in ground state acidity/basicity between azobenzene $E$ and $Z$ isomers has been demonstrated for 2-hydroxyazobenzene, where the $E$ acid is stabilised by hydrogen

Department of Chemistry, Imperial College London, London, SW7 2AZ, UK.

E-mail:m.fuchter@imperial.ac.uk

$\dagger$ Electronic supplementary information (ESI) available. See DOI: 10.1039/ c5cc10380k. Raw data files are openly available, DOI: 10.6084/m9.figshare.2975923 bonding to the azo unit, whereas the $Z$ isomer is not; translating to an increase in acidity of the $Z$ isomer (or conversely an increase in basicity of the deprotonated $E$ isomer). ${ }^{13,14}$ Similarly, the azonium $p$-aminoazobenzenes reported by Woolley and co-workers, exhibit a $\mathrm{p} K_{\mathrm{a}}$ shift of approximately 1.5 units between isomers, due to hydrogen bonding between an ortho oxygen functional group on the azobenzene ring to the protonated azo unit in the $E$ isomer (Fig. 1A). ${ }^{15,17}$ Hydrogen bonding to the azo unit is not the only plausible strategy for modifying azobenzene acidity/basicity however. Hecht and co-workers, for example, have modulated the basicity of piperidine-containing azobenzenes through steric shielding in one isomer over the other (Fig. 1B). ${ }^{8,18,19}$

We have previously reported novel azoheteroaryl photoswitches based on pyrroles and pyrazoles, ${ }^{20}$ where the type, positioning, and substitution of the heteroaryl ring impacts key switching properties such as addressability, efficiency and thermal stability. However the

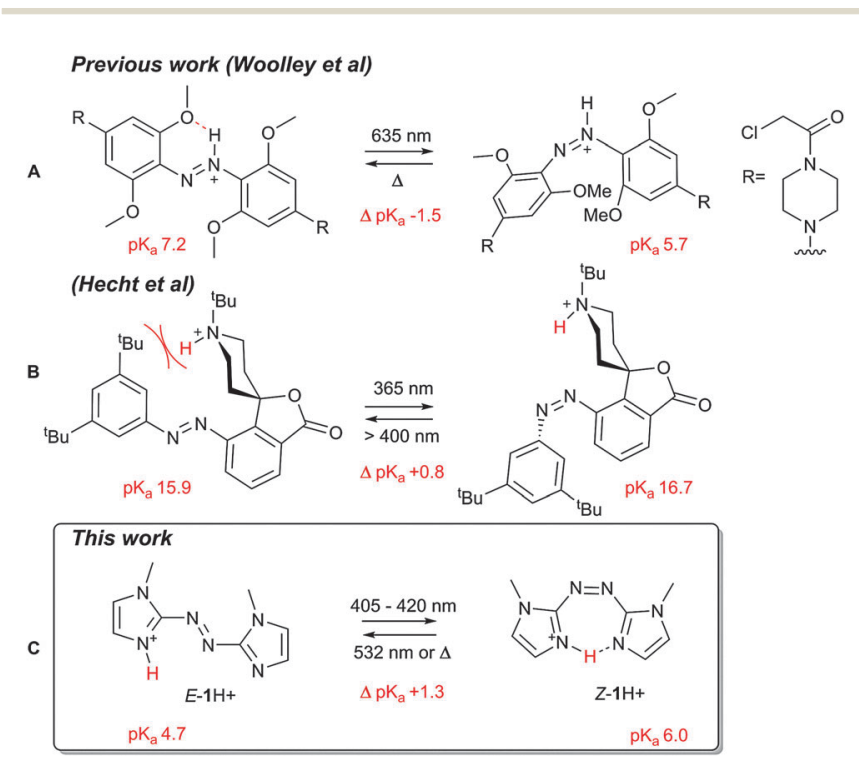

Fig. 1 Previous azo photoswitchable bases reported by (A) Woolley, and; (B) Hecht, and; (C) our work. 
presence of heteroatoms on the heteroaryl ring system presents other functional opportunities.

For example, Herges and co-workers have used the pyridine nitrogen of azopyridines as a ligating unit to access photodissociable ligands, ${ }^{3,21,22}$ and have recently demonstrated an improvement in ligand affinity upon moving to arylazoimidazoles. ${ }^{23}$ Herein we report the first photoswitchable Brønsted base of this class, utilising an azobisimidazole. Importantly with respect to the state of the art (Fig. 1), the protonation event occurs on one of the imidazole rings and is stabilised by the neighbouring ring, rather than through participation of the azo moiety. This results in a compound in which the $Z$ isomer is over an order of magnitude more basic than the $E$ isomer. Furthermore, by avoiding protonation of the azo linkage, the thermal half-life of the protonated $Z$ isomer is increased relative to the neutral form.

Imidazole was chosen as the heteroaromatic ring for this study, due to its high basicity relative to other heteroarenes. Both azobis(2-imidazole) 1 and azobis(4-imidazole) 2 (see Fig. 2) were considered to have the correct connectivity to enable proton stabilisation in the $Z$ isomer. Energy minimised conformations and calculated UV/Vis spectra were obtained for both these photoswitches in their neutral states using B3LYP/6-31G(d,p) and CAM-B3LYP/6-311G(2df,2p) respectively (Fig. 2). Azobisimidazole 1, which has the azo moiety attached at the 2-position of both imidazole moieties, was calculated to have a large $\mathrm{n}-\pi^{*}$ absorbance in the $Z$ isomer, and hence good separation of absorbances between the $E$ and $Z$ isomers, which should allow for excellent photoconversion between isomers at different wavelengths (good addressability ${ }^{24}$ ). This large $n-\pi^{*}$ absorbance is due to the twisted conformation adopted by the $Z$ isomer (Fig. 2B), which minimises unfavourable steric interactions between a given $\mathrm{N}$-methyl and the neighbouring azoaryl ring, and unfavourable lone pair interactions between the basic imidazole nitrogens and the azo nitrogens. Conversely, the energy minimised conformation of 2 predicts a planar conformation for the $Z$ isomer and hence a negligible $n-\pi^{*}$ absorbance is predicted on symmetry grounds (Fig. 2B). These calculations are consistent with our findings on the conformational preferences and spectroscopic properties of the arylazopyrazoles and arylazopyrroles ${ }^{20}$ - when there is a mirror plane through the molecule, as with the planar $E$ isomers and $Z-2$, the $\mathrm{n}-\pi^{*}$ transition is symmetry forbidden and no absorbance is predicted (although experimentally a weak absorbance is seen due to vibrational and rotational motion). The calculated spectrum for $E-1$ also had a significantly red-shifted $\pi-\pi^{*}$ absorbance compared to $E-2$,

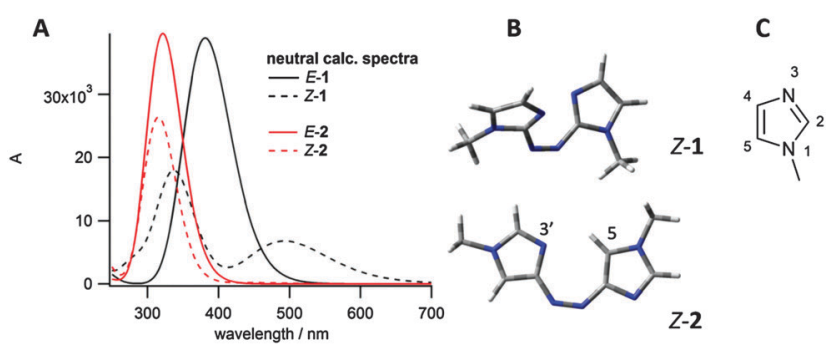

Fig. 2 (A) Calculated spectra for neutral 1 and 2 using CAM-B3LYP/ $6-311 G(2 d f, 2 p)$ level of theory; (B) energy minimised conformations of Z-1 and Z-2 using B3LYP/6-31G(d,p); (C) numbering of the imidazole ring.
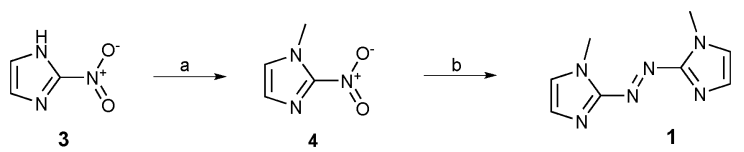

Scheme 1 Reagents and conditions: (a) Mel, $\mathrm{K}_{2} \mathrm{CO}_{3}, \mathrm{MeCN}, 0-65{ }^{\circ} \mathrm{C}$, $65 \%$; (b) $\mathrm{NH}_{4} \mathrm{Cl}$, Zn dust, $\mathrm{H}_{2} \mathrm{O}, 45^{\circ} \mathrm{C}, 49 \%$.

suggesting that visible light rather than UV light could be used for photoswitching. On the basis of these two features, azobisimidazole 1 was chosen for further study. Energy minimised conformations of protonated 1 confirmed that in the $Z$ isomer, the proton would be stabilised by interacting with both basic nitrogens in the photoswitch (as shown in Fig. 4B) resulting in a planar conformation and, in turn, a decreased $n-\pi^{*}$ absorbance (Fig. 4A).

Azobisimidazole 1 was prepared in two steps from commercially available 2-nitroimidazole 3 (Scheme 1). First, $N$-methylation was performed using methyl iodide to give compound 4. In addition to impacting the conformation of these systems (vide supra), $N$-methylation of azole azoheteroaryl photoswitches is required to avoid very fast thermal isomerisation of the $Z$ isomer through tautomerisation..$^{25,26}$ Reductive coupling of $\mathbf{4}$ using zinc in aqueous ammonium chloride, ${ }^{27}$ gave the required photoswitch $\mathbf{1}$.

Irradiation was initially carried out on the neutral compound $\mathbf{1}$ in aqueous buffer at $\mathrm{pH} 9$ (where $\mathbf{1}$ was assumed to be not-significantly protonated; this was later shown to be the case, vide infra) using a range of wavelengths (Fig. 3A). The best $E-Z$ photoconversion was achieved using a $408 \mathrm{~nm}$ laser diode, with an estimated photostationary state (PSS) of (90 \pm 3$) \% Z$ (see ESI, $\dagger$ for estimation); however $415 \mathrm{~nm}$ irradation (generated by a dye laser), with an PSS of $(79 \pm 5) \% Z$, was chosen to carry out further photoswitching, due to the tunability of the power using this light source. $\$ 532 \mathrm{~nm}$ irradiation of the $n-\pi^{*}$ absorbance of the $Z$ isomer resulted in quantitative switching of the azobisimidazole back to the

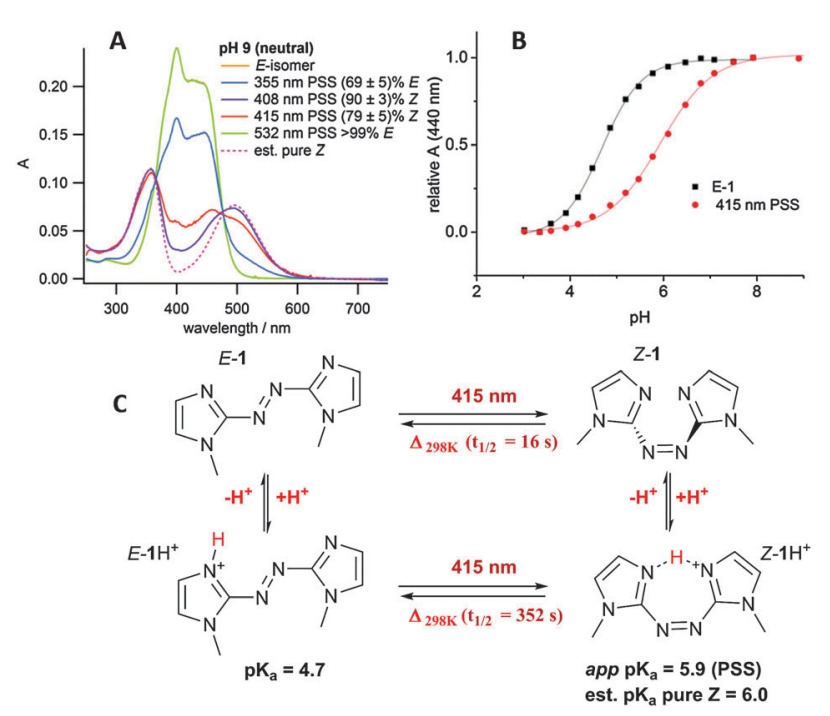

Fig. 3 (A) UV/vis spectra of the different PSS of 1 at different irradiation wavelengths, and estimated pure $Z$ spectrum (at $10^{-5} \mathrm{M}$ ); (B) pH titration of $E-1$ and the $415 \mathrm{~nm}$ PSS; (C) the photoswitch species present at different $\mathrm{pHs}$ and irradiation wavelengths. Thermal isomerisation rates and $\mathrm{p} K_{\mathrm{a}}$ values are included. 
$E$ isomer. $\S$ Notably, $Z-1$ has an intense $n-\pi^{*}$ absorbance $\left(\lambda_{\max }=\right.$ $495 \mathrm{~nm}, \varepsilon=8000 \mathrm{M}^{-1} \mathrm{~cm}^{-1}$ ), much stronger than that seen for $Z$ azobenzenes $\left(\lambda_{\max }=\sim 450 \mathrm{~nm}, \varepsilon=1500 \mathrm{M}^{-1} \mathrm{~cm}^{-1}\right) .{ }^{28}$ This is in line with the DFT calculations that predict a twisted conformation (vide supra). The thermal isomerisation rate of neutral 1 was measured at $25{ }^{\circ} \mathrm{C}$ using UV/vis spectrometry in aqueous buffer (at $\mathrm{pH}$ 9) and the half-life was found to be 16 seconds. It is interesting that the $Z$ isomer of the unsymmetrical azole photoswitch phenylazo-2-imidazole has been previously reported to have a thermal half-life of 9 hours (albeit in a different solvent). ${ }^{29}$ Clearly, the move to a symmetric bisimidazole azo compound has a large impact on the energetics of thermal stability in imidazole-based photoswitches. The origins of thermal stability in such heteroaromatic azo compounds is under further study and will be reported in due course.

In order to determine the $\mathrm{p} K_{\mathrm{a}}$ of both the $E$ isomer and the $415 \mathrm{~nm}$ PSS (79\% $Z$ ), a pH titration was carried out on a buffered aqueous sample of 1 . At each $\mathrm{pH}$ increment the UV/vis spectra of the $E$ isomer and PSS were measured. Due to the reasonably fast thermal isomerisation rate, the irradiated sample was allowed to thermally isomerise back to the $E$ isomer before adjusting the $\mathrm{pH}$. The $\pi-\pi^{*}$ absorption maximum of the $E$ isomer at $440 \mathrm{~nm}$ (Fig. 3A) was plotted against $\mathrm{pH}$ in order to obtain the corresponding titration curves (Fig. 3B). The $\mathrm{pH}$ titration confirmed that at pH 9 azobisimidazole 1 was indeed neutral. The $\mathrm{p} K_{\mathrm{a}}$ was found to be 4.7 for the $E$ isomer and the apparent $\mathrm{p} K_{\mathrm{a}}$ was 5.9 for the $E / Z$ mixture present in the $415 \mathrm{~nm}$ PSS. By considering the PSS and the equilibria present in the system (Fig. 3C), the $\mathrm{p} K_{\mathrm{a}}$ of the $Z$ isomer was estimated as 6.0 (see $\mathrm{ESI} \dagger$ ). A rough titration of the $E$ isomer showed a second protonation event (assigned to protonation of the second imidazole ring) with an approximate $\mathrm{p} K_{\mathrm{a}}$ of 1.6 (see ESI $\dagger$ ). No second protonation event was observed for the $Z$ isomer above $\mathrm{pH} 3$.

Given this data, the UV/vis spectrum for $E-\mathbf{1}$ was recorded in acidic aqueous buffer at $\mathrm{pH} 3$, to measure the photoswitch parameters of the protonated compound $1 \mathrm{H}^{+}$. Irradiation at $415 \mathrm{~nm}$ led to a PSS with a very similar spectrum to that of the $E$ isomer (Fig. 4A). Although at first this could be considered to be a result of a PSS containing predominantly the $E$ isomer, we instead attribute it to the fact that the protonated $E$ and $Z$ isomers $E-\mathbf{1} \mathrm{H}^{+}$and $Z-\mathbf{1} \mathrm{H}^{+}$have very similar $\mathrm{UV} /$ vis spectra. This assignment is supported by TDDFT calculated spectra for the protonated $E$ and $Z$ isomers (Fig. 4A); the spectra of both

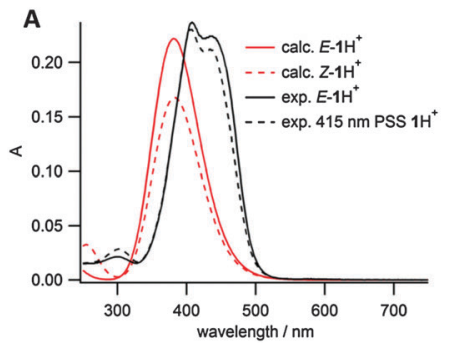

B

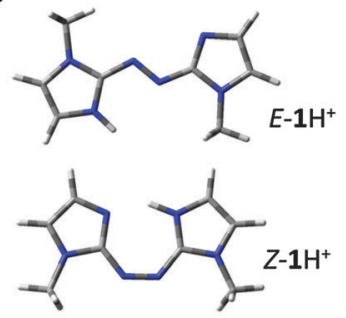

Fig. 4 (A) Comparison of experimental and calculated (CAM-B3LYP//6$311 \mathrm{G}(2 \mathrm{df}, 2 \mathrm{p})) \mathrm{E}-\mathbf{1} \mathrm{H}^{+}$and $Z-1 \mathrm{H}^{+}$spectra; (B) energy minimised conformations (B3LYP/6-31G(d,p)) of $E-1 \mathrm{H}^{+}$and $Z-1 \mathrm{H}^{+}$. isomers is highly similar due to their planar conformation (Fig. 4B), as opposed to the neutral compound 1 (vide supra). As was the case for the neutral compound $\mathbf{1}$, the protonated compound $1 \mathrm{H}^{+}$could be switched between two PSS upon irradiation with 415 and $532 \mathrm{~nm}$ light, however due to the similarity in the $E$ and $Z$ spectra, the PSS compositions could not be obtained. $\uparrow$ The thermal isomerisation rate of protonated $1 \mathrm{H}^{+}$at $25{ }^{\circ} \mathrm{C}$ was found to be approximately 20 -fold slower than for the neutral compound in aqueous buffer $\left(t_{1 / 2}=352 \mathrm{~s}\right.$ for protonated $v s .16 \mathrm{~s}$ for neutral). This increased half-life of the $Z$ isomer on protonation is attributed to the requirement to (partially) break the intramolecular hydrogen bond present in $Z-\mathbf{1} \mathrm{H}^{+}$in either the rotation or inversion transition states for thermal $Z-E$ isomerisation. This trend is opposite to that observed by Woolley and co-workers for their tetra-o-methoxy substituted aminoazobenzene photoswitches upon protonation (Fig. 1A); where the protonated $Z$ isomer has a much shorter half life than the neutral species. ${ }^{15,17}$ This difference is explained by the site of protonation for the different photoswitches; protonation of the azo linkage in the substituted aminoazobenzenes (Fig. 1A) increases the thermal $Z-E$ isomerisation rate, whereas in our case, protonation of 1 does not involve participation of the azo linkage.

Energy minimised conformations of $E-\mathbf{1 H}^{+}$support our assumption that protonation occurs on the imidazole nitrogen rather than the azo. The global minimum for the imidazolium is significantly lower energy than for the azonium ( $\Delta G$ (azonium imidazolium) $=44.4 \mathrm{~kJ} \mathrm{~mol}^{-1}-$ see ESI $\left.\dagger\right)$. In order to further characterise the protonated compounds, the carbon NMR chemical shifts $\left(\delta_{\exp }\right)$ for deuteronated $E$-1 were compared to calculated chemical shifts $\left(\delta_{\text {calc }}\right)$ for $E-\mathbf{1} \mathrm{H}^{+}$protonated on either the imidazole or the azo (see ESI $\dagger$ ). The difference in chemical shift between $\delta_{\text {exp }}$ and $\delta_{\text {calc }}$ was much smaller for the imidazolium calculated structure than the azonium. Experimentally, $\mathrm{p} K_{\mathrm{a}}$ values for azonium cations are generally several units lower than imidazolium cations, ${ }^{30-33}$ with the exception being when the azonium proton can be additionally stabilised by hydrogen bonding as in the systems reported by Woolley and co-workers (e.g. Fig. 1A). ${ }^{15,17}$ Together the computational and experimental data supports our assumption that the protonation occurs on the imidazole rather than the azo nitrogen.

Given the significant $\mathrm{p} K_{\mathrm{a}}$ difference between the $E$ and $Z$ isomers of azobisimidazole $\mathbf{1}$, we sought to achieve a reversible pH 'jump' upon photoswitching. In principle, switching solution $\mathrm{pH}$ could have many uses, such as controlling the rate of a specific base catalysed reaction, or modulating enzyme activity. ${ }^{10}$ In order to measure the $\mathrm{pH}$ of the solution a $\mathrm{pH}$ indicator was used. ${ }^{34}$ Bromocresol purple (BCP) was chosen as it has a similar $\mathrm{p} K_{\mathrm{a}}$ value (vide infra) to that of the photoswitch, and it has an absorption maximum at a wavelength where the azobisimidazole has a negligible absorbance. A pH titration was carried out on BCP (Fig. S5, ESI $\dagger$ ) and the $\mathrm{p} K_{\mathrm{a}}$ value obtained (6.8) was similar to the literature value of $6.2-6.4 .^{35}$ Prior to attempting the $\mathrm{pH}$ 'jump', $\mathrm{pH}$ titrations of both $E-\mathbf{1}$ and the $405 \mathrm{~nm}$ PSS with equimolar BCP were carried out (Fig. 5A). From this a graph of $A_{\max }(\mathbf{1}) / A_{\max }(\mathrm{BCP})$ against $\mathrm{pH}$ was plotted (for both $E$ isomer and PSS) and the exponential fit obtained (Fig. S6, ESI $\dagger$ ). Using this graph the $\mathrm{pH}$ value could be extracted 

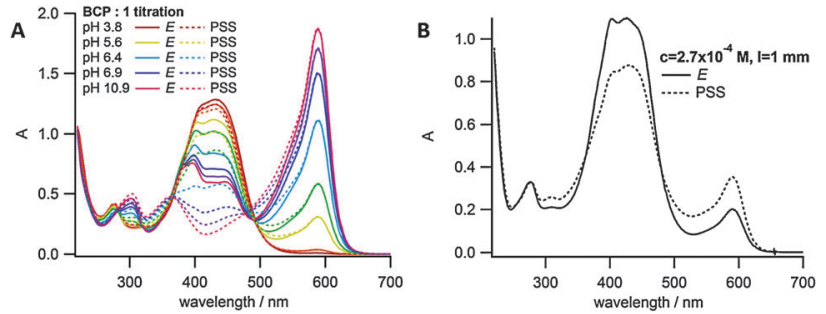

Fig. $5 \quad E-1$ and $405 \mathrm{~nm}$ PSS with equimolar BCP; (A) pH titration; (B) $\mathrm{pH}$ 'jump' experiment at $2.7 \times 10^{-4} \mathrm{M}, 1 \mathrm{~mm}$ path length.

from the UV/vis spectrum of a given sample of $\mathbf{1}$ and equimolar BCP. Irradiation of a $2.7 \times 10^{-4} \mathrm{M}$ aqueous solution of 1 at $405 \mathrm{~nm}$ led to an immediate change in $\mathrm{pH}$ (Fig. 5B). A change of $0.29 \mathrm{pH}$ units was observed, equating to a two-fold reduction in proton concentration in the PSS. In theory, using a 1:1 mixture of $E-\mathbf{1}$ and $E-1 \mathrm{H}^{+}$should enable a pH jump equal to the $\Delta \mathrm{p} K_{\mathrm{a}}$ of the photoswitch (approximately 1.3 in this case). However, limitations on the concentrations used in this experiment and buffering by the indicator and potentially by carbonate is likely to decrease the range that can be obtained. Decreasing the concentration from $10^{-4}$ to $10^{-5} \mathrm{M}$ resulted in a decrease in the $\mathrm{pH}$ 'jump' observed. While an increase in concentration would be expected to result in an increase in the $\mathrm{pH}$ jump, we were unable to assess this due to limitations in the method used for $\mathrm{pH}$ detection and the fast thermal isomerisation rates of the photoswitch. Nonetheless, several repeated cycles of photoswitching were carried out to ensure that the $\mathrm{pH}$ jump was reproducible over multiple irradiation cycles (Fig. S7, ESI + ). The pH jump was consistent each time, within 3\% of the mean value, suggesting that no irreversible photochemistry involving the indicator was occurring.

In conclusion, we have designed an azoheteroaryl photoswitch that bears basic nitrogens on the two heteroaryl rings, correctly positioned to stabilise a proton in the $Z$ isomer. This results in a significant and reversible shift in $\mathrm{p} K_{\mathrm{a}}$ values between the $E$ and $Z$ isomers. We believe extension of the concept developed in this paper may lead to related photochromic molecules with an increased difference in $\mathrm{p} K_{\mathrm{a}}$ between the isomers, and that such systems may lead to a wide range of applications. For example, photoswitchable bases have already been shown to have potential as switchable general base catalysts, ${ }^{8}$ but significant basicity differences between isomers may open up the possibility of switchable specific base catalysis and enzyme catalysis. Furthermore, light-driven proton concentration oscillations ${ }^{14}$ may be used to extract kinetic data on proton transfer events in a range of chemical and biological systems.

We thank the Engineering and Physical Sciences Research Council and the Leverhulme Trust (RPG-2012-441 to R. D. R.) for funding. We also thank Dr Kuimova for equipment access.

\section{Notes and references}

\$ When $Z-E$ thermal isomerisation is fast, higher power is needed to reach the PSS.
$\S$ This occurred at a faster rate than that of the $Z-E$ thermal isomerisation.

I The thermal isomerisation was too fast to allow quantification of the PSS by other spectroscopic methods (for example, by NMR).

1 T. Fehrentz, M. Schönberger and D. Trauner, Angew. Chem., Int. Ed., 2011, 50, 12156-12182.

2 W. A. Velema, W. Szymanski and B. L. Feringa, J. Am. Chem. Soc., 2014, 136, 2178-2191.

3 S. Thies, H. Sell, C. Bornholdt, C. Schütt, F. Köhler, F. Tuczek and R. Herges, Chem. - Eur. J., 2012, 18, 16358-16368.

4 T. Hugel, N. B. Holland, A. Cattani, L. Moroder, M. Seitz and H. E. Gaub, Science, 2002, 296, 1103-1106.

5 W. R. Browne and B. L. Feringa, Nat. Nanotechnol., 2006, 1, 25-35.

6 K. Matsuda and M. Irie, Polyhedron, 2005, 24, 2477-2483.

7 M. Alemani, M. V. Peters, S. Hecht, K. H. Rieder, F. Moresco and L. Grill, J. Am. Chem. Soc., 2006, 128, 14446-14447.

8 M. V Peters, R. S. Stoll, A. Kühn and S. Hecht, Angew. Chem., Int. Ed., 2008, 47, 5968-5972.

9 M. Samanta, V. S. R. Krishna and S. Bandyopadhyay, Chem. Commun., 2014, 50, 10577-10579.

10 S. Kohse, A. Neubauer, A. Pazidis, S. Lochbrunner and U. Kragl, J. Am. Chem. Soc., 2013, 135, 9407-9411.

11 S. Abbruzzetti, M. Carcelli, D. Rogolino and C. Viappiani, Photochem. Photobiol. Sci., 2003, 2, 796-800.

12 S. H. Kawai, S. L. Gilat and J. Lehn, Eur. J. Org. Chem., 1999, 2359-2366.

13 M. Emond, J. Sun, J. Grégoire, S. Maurin, C. Tribet and L. Jullien, Phys. Chem. Chem. Phys., 2011, 13, 6493-6499.

14 M. Emond, T. Le Saux, S. Maurin, J.-B. Baudin, R. Plasson and L. Jullien, Chem. - Eur. J., 2010, 16, 8822-8831.

15 S. Samanta, A. Babalhavaeji, M.-X. Dong and G. A. Woolley, Angew. Chem., Int. Ed., 2013, 52, 14127-14130.

16 N. Abeyrathna and Y. Liao, J. Am. Chem. Soc., 2015, 137, 11282-11284.

17 M. Dong, A. Babalhavaeji, M. J. Hansen, L. Kalman and G. A. Woolley, Chem. Commun., 2015, 51, 12981-12984.

18 R. S. Stoll and S. Hecht, Org. Lett., 2009, 11, 4790-4793.

19 R. S. Stoll, M. V Peters, A. Kuhn, S. Heiles, R. Goddard, M. Buhl, C. M. Thiele and S. Hecht, J. Am. Chem. Soc., 2009, 131, 357-367.

20 C. Weston, R. Richardson, P. Haycock, A. White and M. Fuchter, J. Am. Chem. Soc., 2014, 136, 11878-11881.

21 F. Matino, G. Schull, U. Jana, F. Köhler, R. Berndt and R. Herges, Chem. Commun., 2010, 46, 6780-6782.

22 S. Thies, H. Sell, C. Schütt, C. Bornholdt, C. Näther, F. Tuczek and R. Herges, J. Am. Chem. Soc., 2011, 133, 16243-16250.

23 C. Schütt, G. Heitmann, T. Wendler, B. Krahwinkel and R. Herges, J. Org. Chem., 2016, 81, 1206-1215.

24 D. J. van Dijken, P. Kovaříček, S. P. Ihrig and S. Hecht, J. Am. Chem. Soc., 2015, 137, 14982-14991.

25 J. Otsuki, K. Suwa, K. K. Sarker and C. Sinha, J. Phys. Chem. A, 2007, 111, 1403-1409.

26 J. Chen and Z. Yin, Dyes Pigm., 2014, 102, 94-99.

27 E. Gattavecchia and D. Tonelli, J. Chem. Soc., Perkin Trans. 2, 1986, 689-693.

28 H. M. D. Bandara and S. C. Burdette, Chem. Soc. Rev., 2012, 41, 1809-1825.

29 J. Otsuki, K. Suwa, K. Narutaki, C. Sinha, I. Yoshikawa and K. Araki, J. Phys. Chem. A, 2005, 109, 8064-8069.

30 S. Stoyanov, L. Antonov, T. Stoyanova and V. Petrova, Dyes Pigm., 1996, 32, 171-185.

31 T. Stoyanova, S. Stoyanov, L. Antonov and V. Petrova, Dyes Pigm., 1996, 31, 1-12.

32 A. A. M. Prabhu, G. Venkatesh, R. K. Sankaranarayanan, S. Siva and N. Rajendiran, Indian J. Chem., Sect. A: Inorg., Bio-inorg., Phys., Theor. Anal. Chem., 2010, 49, 407-417.

33 J. A. Dean, Lang's Handbook of Chemistry, McGraw-Hill, New York, 12th edn, 1979.

34 R. M. D. Nunes, M. Pineiro and L. G. Arnaut, J. Am. Chem. Soc., 2009, 131, 9456-9462.

35 R. W. Sabnis, Handbook of acid-base indicators, CRC Press, Boca Raton, 2007. 